\title{
Central cholinergic neuronal degeneration promotes the development of postoperative cognitive dysfunction
}

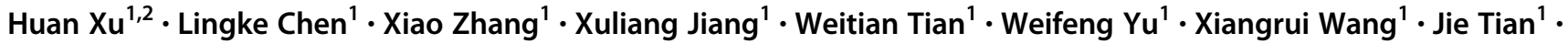 \\ Diansan $\mathrm{Su}^{1}$
}

Received: 10 May 2018 / Revised: 11 October 2018 / Accepted: 7 November 2018 / Published online: 9 January 2019

(c) United States \& Canadian Academy of Pathology 2019

\begin{abstract}
Postoperative cognitive dysfunction (POCD) is consistently associated with increased morbidity and mortality. However, its mechanism remains poorly understood. We hypothesized that central cholinergic neuronal degeneration facilitates the development of POCD. The impact of anesthesia/surgery (appendectomy) on learning and memory and the levels of choline acetyltransferase (ChAT), acetylcholinesterase (AChE), vesicular acetylcholine transporter (VAChT), and choline transporter (CHT) in adult and aged mice were measured. Separate cohorts were analyzed after pretreatment with donepezil, an AChE inhibitor, in aged mice or with murine-p75-saporin (mu-p75-sap), a cholinergic-specific immunotoxin, in adult mice. Morris Water Maze was used to measure the learning and memory changes after anesthesia/surgery. Western blot was used to measure the changes in the protein levels of the biomarkers of the central cholinergic system. We found that anesthesia/surgery-induced memory decline and attenuation of central cholinergic biomarkers (ChAT and VAChT) in aged mice but not in adult mice. Donepezil pretreatment reduced central cholinergic impairment in the aged mice and prevented learning and memory declines after anesthesia/surgery. In contrast, when central cholinergic neurons were pre-injured with mu-p75-sap, cognitive dysfunction developed in the adult mice after anesthesia/surgery. These data suggest that central cholinergic neuronal degeneration facilitates the development of POCD.
\end{abstract}

\section{Introduction}

Postoperative cognitive dysfunction (POCD) commonly occurs after anesthesia and surgery [1]. Deficits in cognitive functions, including learning and memory, are the major clinical features of POCD [2]. The reported incidence of POCD has ranged from 25 to $53 \%$ [3, 4]. Accumulating

These authors contributed equally: Huan Xu, Lingke Chen.

Supplementary information The online version of this article (https:// doi.org/10.1038/s41374-018-0174-9) contains supplementary material, which is available to authorized users.

$\triangle$ Jie Tian

Vaseline2001@ hotmail.com

$\triangle$ Diansan $\mathrm{Su}$

diansansu@yahoo.com

1 Department of Anesthesiology, Renji Hospital, School of Medicine, Shanghai Jiaotong University, Shanghai 200127, China

2 Department of Anesthesiology, Shanghai Pulmonary Hospital, Tongji University, Shanghai 200433, China evidence suggests that POCD is associated with increased post-surgical mortality, disability, and early retirement [5]. Thus, POCD has become a major concern among anesthesiologists, surgeons, and their patients.

Despite an increasing number of studies that have explored cellular and molecular alterations in POCD, mechanisms remain elusive. Advanced age is now accepted as the primary risk factor of POCD, which is supported by a growing body of clinical evidence [6] and animal studies [7-9]. The central cholinergic system, which plays an important role in learning and memory [10-12], may begin to degenerate in early presymptomatic stages of neurodegenerative disease [13]. We hypothesize that the level of central cholinergic neuronal degeneration before surgery may influence the development of POCD.

The present study was carried out to investigate a possible role of central cholinergic neuronal degeneration in the development of POCD. 


\section{Material and methods}

This study was approved by the Shanghai Jiaotong University School of Medicine Animal Care and Use Committee. All the procedures were conducted in accordance with the guidelines of the National Institutes of Health for animal care. Every effort was made to minimize animal suffering and the number of animals used.

\section{Animals}

Adult (two-month-old) and aged (eighteen-month-old) C57BL/6 mice were provided by the Sino-British SIPPR/ BK Lab (Shanghai, China). All the mice were housed in groups of 3-5 mice per cage under controlled conditions with temperature $\left(22 \pm 1{ }^{\circ} \mathrm{C}\right)$ and humidity level $(50 \pm 5 \%)$. The lights were maintained on a $12 \mathrm{~h}$ light-dark cycle. All the mice had free access to standard rodent diet and water. Body weights of all mice were recorded daily during the experiment, and no significant differences were detected among the experimental groups throughout the procedure. The mice were randomly assigned to different groups using SAS software (SAS Institute Inc., Cary, NC).

\section{Experimental protocol}

As shown in supplemental Fig. 1, three series of experiments were included in the present study. Behavioral changes in adult and aged mice were measured with the Morris Water Maze (MWM) starting at 2 days after surgery and continuing for 5 consecutive days. To improve the function of central cholinergic systems of the aged mice, donepezil was administered for 28 consecutive days before surgery in one set of aged mice. To injibit the central cholinergic system selectively, a single injection of mu-p75sap, an immunotoxin, which is specific for cholinergic neurons, was injected intracerebroventricularly in another set of normal adult mice 20 days before appendectomy. Behavioral changes were measured by the MWM 2 days after appendectomy in all mice for 5 consecutive days. The expression levels of the cholinergic biomarkers ChAT, $\mathrm{AChE}$, vesicular acetylcholine transporter (VAChT), and choline transporter (CHT) were measured by western blot or immunohistochemical staining.

\section{Surgery}

To minimize the effects of anesthesia, neuroleptic analgesia [fentanyl $(200 \mu \mathrm{g} / \mathrm{kg})$ and droperidol $(10 \mathrm{mg} / \mathrm{kg})$ (i.p.)] was used in the current study [14]. According to a previous study, this regimen can provide sufficient sedation and pain relief for rodents during surgery without affecting their learning ability or memory [15].
Mice underwent a standard surgical procedure for appendectomy as described previously [16]. Firstly, a midline laparotomy ( $1 \mathrm{~cm}$ in length) was performed under fentanyl and droperidol anesthesia, and then mobilization and exteriorization of appendix followed. Two ligatures were placed proximal to the border of the appendix and the cecum, and division of the appendix was conducted between the two ligatures. A single dose of butorphanol $(0.4 \mathrm{mg} / \mathrm{kg}$, s.c.) was administered for postoperative analgesia at the end of surgery. The cecal stump was irrigated with saline, and the two-layer abdominal wall was sutured with 5-0 Vicryl sutures. Aseptic techniques were used during the entire procedure, which required approximately $15 \mathrm{~min}$. The mice were breathing spontaneously and recovered from anesthesia within $20 \mathrm{~min}$. No intubation or supplemental oxygen was needed, and no animals died, including the mice in the aged group. During the procedure, the temperature was maintained between 36 and $37^{\circ} \mathrm{C}$ with the aid of warming pads. $\mathrm{SpO}_{2}$ was monitored during the procedure, and no hypoxia $\left(\mathrm{SpO}_{2}<90 \%\right)$ was observed during the procedure.

\section{Morris water maze}

The changes of learning and memory were measured by the MWM 2 days after surgery according to our previous studies [9]. The MWM included a circular tank $(110 \mathrm{~cm}$ in diameter and $30 \mathrm{~cm}$ in depth) where the mice were trained to escape from the water by swimming to a hidden platform $1.5 \mathrm{~cm}$ beneath the surface. The pool was surrounded by curtains, and the location of the platform could only be identified by distal extra-maze cues attached to the curtains. The water temperature was maintained at $23-25^{\circ} \mathrm{C}$ and was made opaque with nontoxic white paint throughout all of the training and testing days. The pool was divided into four quadrants: target, opposite, adjacent 1 , and adjacent 2 . A camera connected to a video recorder and a computerized tracking system were used to record the experiments.

The MWM test was a 5 day procedure. The first 4 days represented the reference memory test phase and totally 16 training trials included: 4 training trials in each training day with an inter-trial interval of 30-40 min. For each trail, mouse was released into water from one of the four quadrants facing the wall. The order of starting point was randomly changed every day. But the order of starting point on each training day was fixed. Each mouse was allowed to find and mount the platform within $60 \mathrm{~s}$. And the mouse was allowed to stay on the platform for $20 \mathrm{~s}$, in order to remember the spatial information. Then the mouse was removed and was placed in a holding cage with a heating lamp to keep warm. If the mouse failed to locate and mount the platform within $60 \mathrm{~s}$, the mouse was gently guided to the platform and was required to stay on the platform for $20 \mathrm{~s}$ 
before transferred to the holding cage. A video camera mounted above the pool was used to track the mice spent to find and mount the platform (escape latency); the time spent in each quadrant and the swimming speed were calculated by MWM software (Shanghai Jiliang Software Technology Co. Ltd., China) according to the recorded videos. A probe test was performed on the fifth day. In the probe test, the platform was removed from the water tank, and the mice were allowed to swim freely for $60 \mathrm{~s}$. The amount of time spent in the target quadrant was recorded and calculated. A person who was blinded to the group assignments performed the MWM.

\section{Western blot assays}

The whole hippocampus of each mouse was harvested immediately after the MWM test. The harvested tissues were homogenized in RIPA lysis with a mixture of phosphatase/ protease inhibitors, and centrifugation at $12,000 \times g$ for 15 min at $4{ }^{\circ} \mathrm{C}$ following. The supernatants were collected, and the protein concentrations of the samples were determined using a bicinchoninic acid (BCA) protein assay kit (Thermo, USA). The protein in supernatants was dissolved in $5 \times$ sample loading buffer and denatured at $100^{\circ} \mathrm{C}$ for $10 \mathrm{~min}$. The proteins $(50 \mu \mathrm{g})$ were electrophoresed by SDS-PAGE gel, and the separated proteins were transferred to polyvinylidene difluoride (PVDF) membranes (pore size, 0.45 $\mu \mathrm{m}$, Millipore, USA). The membranes were blocked for $1 \mathrm{~h}$ with 5\% milk in Tris-buffered saline and Tween-20 (TBST) at room temperature. And the membranes were incubated with anti-ChAT antibody (1:2000, Abcam, Cambridge, MA), anti-AChE antibody (1:500, Santa Cruz Biotechnology, Santa Cruz, CA), anti-VAChT antibody (1:1000, Abcam, Cambridge, UK), anti-CHT antibody (1:500, Pierce, Rockford, IL), and anti- $\beta$-actin antibody (1: 10,000, Cell Signaling, Danvers, MA) in TBST plus $5 \%$ bovine serum albumin overnight at $4{ }^{\circ} \mathrm{C}$. An appropriate horseradish peroxidase (HRP)-conjugated secondary antibodies (anti-rabbit or anti-mouse; 1:5000, Cell Signaling) were used to incubate the membranes at room temperature for $1 \mathrm{~h}$. After that, the proteins were detected with enhanced chemiluminescence (ECL) system (Pierce). The intensity of the western blot signals was quantified using a ChemiDoc ${ }^{\mathrm{TM}} \mathrm{XRS}+$ system (Bio-Rad, USA) and was expressed as a ratio relative to $\beta$ actin protein [17]. Based on previous experience, we set $n=$ 4 in the western blot experiment [18].

\section{Immunohistochemistry}

After the MWM, 4 mice in each group were anesthetized by pentobarbital sodium. Then they were transcardially perfused with cooled $0.9 \%$ sodium chloride, followed by perfused with cooled paraformaldehyde (4\%). A microtome was used to cut paraffin wax-embedded brain by coronal sections $(10 \mu \mathrm{m})$ at the level of the basal forebrain. Immunohistochemistry was then performed according to the manufacturer's manual. Briefly, the tissue sections were dewaxed and rehydrated firstly, and then microwave oven was used to immerse and boil the sections in citrate buffer ( $\mathrm{pH}$ 8.0) for antigen retrieval. After pre-blocked with normal goat serum, the sections were incubated with antiChAT antibody (1:500; Abcam, Cambridge, UK). The percentage of the positive area was determined using ImageJ software (rsbweb.nih.gov).

\section{Immunofluorescence}

After completing the MWM, 4 mice in each group were anesthetized by pentobarbital sodium. Then they were transcardially perfused with $0.9 \%$ sodium chloride, followed by cooled paraformaldehyde (4\%). After post-fixation and dehydration, brains were embedded in O.C.T. compound (Tissue-Tek, Torrance, CA) on dry ice. O.C.T-embedded frozen brain tissue sections were cryosectioned at a thickness of $14-18 \mu \mathrm{m}$ and subsequently fixed in ice cold acetone. Sections (basal forebrain) were blocked with $5 \%$ normal swine serum (Vector Laboratories) for $60 \mathrm{~min}$ and incubated in anti-ChAT antibody (1:100; Abcam, Cambridge, UK) diluted in blocked solution overnight at $4{ }^{\circ} \mathrm{C}$. Slices were washed in PBS and incubated with fluorophore-conjugated secondary antibodies. Sections were washed and mounted in Vectashield mounting medium with DAPI (Vector Laboratories, RRID:AB_2336790). The sections were examined using a confocal microscope (OLYMPUS FV3000).

\section{Interventions}

\section{Improvement of the central cholinergic system in aged mice}

Donepezil is an inhibitor of cholinesterase, which can easily pass the BBB, which is currently used as a treatment for Alzheimer's disease. As an inhibitor of cholinesterase, donepezil significantly increases central acetylcholine level. A group of aged mice received $5 \mathrm{mg} / \mathrm{kg}$ of donepezil (Pfizer, NY) for four weeks before surgery via intragastric administration with a feeding needle in normal saline every day. Mice in the control group received the same amount of normal saline via oral gavage. After oral administration, donepezil can be absorbed easily, and the maximum plasma concentration is reached at 2.4 to $4.4 \mathrm{~h}$. After repeated administrations of donepezil, it takes 21-28 days for plasma levels to reach a steady state [19]. Moreover, previous studies indicated that $5 \mathrm{mg} / \mathrm{kg} / \mathrm{day}$ of donepezil is the optimal dosage in rodent models [20, 21]. Therefore, $5 \mathrm{mg} /$ $\mathrm{kg} / \mathrm{day}$ for 4 weeks was utilized in the present study. 


\section{Pharmacological injury of the central cholinergic system in adult mice}

Murine-p75-saporin (mu-p75-sap) is a commonly used immunotoxin that is specific for cholinergic neurons and can induce selective lesions of the basal forebrain cholinergic system [22]. The mice were anesthetized with fentanyl $(200 \mu \mathrm{g} / \mathrm{kg})$ and droperidol $(10 \mathrm{mg} / \mathrm{kg})$. Then, one microliter of mu-p75-sap (Advanced Targeting Systems, $\mathrm{CA}$ ) at a concentration of $0.8 \mu \mathrm{g} / \mu \mathrm{l}$ was injected into the lateral ventricles using a 26-gauge syringe at the coordinates AP- $0.5 \mathrm{~mm}, \mathrm{ML} \pm 1.0 \mathrm{~mm}$, and DV-2.2 $\mathrm{mm}$ relative to the bregma. Bilateral injections of $0.5 \mu \mathrm{l}$ mu-p75-sap into each lateral ventricle were conducted to the mice. The procedure of injection to each side last for $5 \mathrm{~min}$. In order to minimize reflux after the injection, an additional 5 min was needed for the needle to remain in place. The control animals were injected with the same amount of sterile PBS. The animals recovered fully for 20 days before surgery.

\section{Statistical analysis}

Statistical Package for the Social Sciences software, version 20.0, was used for the statistical analyses. One-way ANOVA was used to analyze western blot data. The MWM data were analyzed using two-way ANOVA with repeated measures, followed by a Bonferroni multiple comparison test. Since surgery and different ages were both investigated in this study, a two-way-ANOVA was used to analyze the impact of these two factors on the ChAT protein level. $P<0.05$ was considered statistically significant.

\section{Results}

\section{Anesthesia/surgery impaired learning and memory in aged but not adult mice}

An appendectomy was performed under anesthesia with fentanyl and droperidol in adult and aged mice. The MWM was used to measure the animals' behavioral performance 2 days after anesthesia/surgery. No behavioral changes were identified after anesthesia/surgery in adult mice, including the mean latency (Fig. 1a), the average swimming speed (Fig. 1c), or the swimming time in the target quadrant on the probe test (Fig. 1b). In contrast, anesthesia/surgery significantly impaired the learning and memory of aged mice. As shown in Fig. 1, the mean latency (Fig. 1d) of the anesthesia/surgery group increased $(P<0.05)$, and the swimming time in the target quadrant (Fig. 1e) decreased significantly $(P<0.05)$ compared with that in the control group. Consistent with a previous study [15] by Wan et al., no significant differences were identified between the anesthesia group and the control group (Fig. 1f).

\section{Anesthesia/surgery impaired the biomarkers of the central cholinergic nervous system in aged but not adult mice}

The animals were sacrificed after surgery, and the brains were harvested. The protein levels of cholinergic markers, including ChAT, AChE, VAChT, and CHT in the hippocampus, were measured by western blot to analyze the injury to the cholinergic nervous system following surgery. None of the biomarkers changed significantly after anesthesia/surgery in the adult mice (Fig. 2a-d). In the aged mice, however, both the expression levels of ChAT and VAChT (Fig. 2e, f) decreased significantly after anesthesia/ surgery compared with the control group $(P<0.05)$, whereas AChE and CHT remained unaffected (Fig. 2g, h), indicating that anesthesia/surgery affected the central cholinergic nervous system in aged but not in adult mice. Anesthesia per se had no effects on these cholinergic biomarkers (Fig. 2a-h). In support of the above studies, immunofluorescence analysis showed that basal forebrain cholinergic neurons decreased with aging, and anesthesia/ surgery profoundly reduced cholinergic neurons in the aged mice (Fig. 2i).

\section{Donepezil pretreatment prevented the spatial learning, memory impairment, and cholinergic biomarkers alterations induced by anesthesia/ surgery in aged mice}

Next, donepezil, an inhibitor of AChE approved by the FDA for the treatment of dementia, was used to inhibit ACh breakdown at the synapse. Donepezil easily crosses the blood-brain barrier (BBB) and improves the cognitive behavior of Alzheimer's disease (AD) patients. In the current study, the pretreatment of donepezil in aged mice prevented the anesthesia/surgery-induced decrease in hippocampal ChAT and VAChT levels (Fig. 3a, b). The AChE and CHT levels were not affected by donepezil (Fig. 3c, d).

Behavioral tests indicated that, after donepezil pretreatment, all the aged mice swam normally in the MWM with similar swimming speeds and none of the mice exhibited difficulty in mounting the platform. As shown in Fig. 3, impairments in spatial learning and memory after anesthesia/surgery were prevented by donepezil pretreatment. The mice that were pre-treated with donepezil spent less time finding the platform and spent more time in the target quadrant during the probe test after anesthesia/surgery 
A

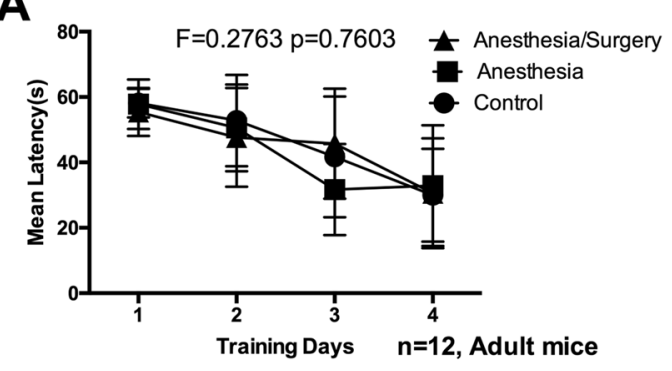

C

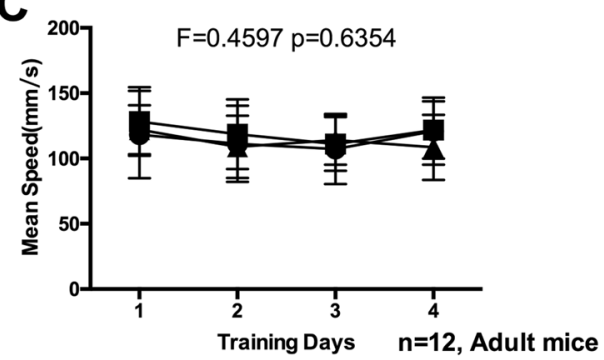

E

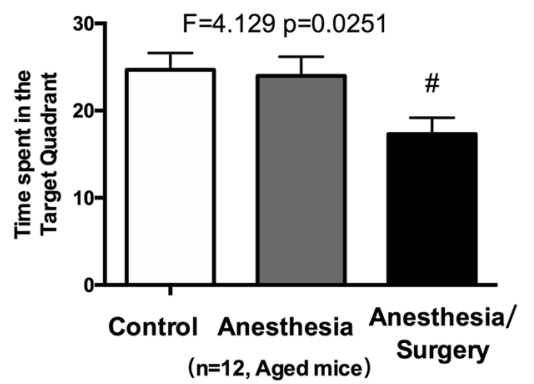

Fig. 1 Anesthesia/surgery impaired learning and memory in aged but not adult mice. a Anesthesia/surgery did not significantly alter the escape latency of mice in MWM test as compared with anesthesia alone or control condition in adult mice. b There was no significant difference in the swimming time spent in the target quadrant on the probe tests in MWM test in the adult mice among anesthesia/surgery, anesthesia and control group. c There was no significant difference in the swimming speed in the MWM test among anesthesia/surgery, anesthesia, and control condition in the adult mice. d The escape latency of aged mice in the anesthesia/surgery group was longer than that of mice either in the anesthesia group or control group. e Time

compared with the mice without donepezil treatment (Fig. 3e, f). No significant changes in the average swimming speed were observed among all the groups (Fig. 3g).

\section{Spatial learning and memory were impaired after anesthesia/surgery in adult mice with central cholinergic systems pre-injured by mu-p75-sap}

To further elucidate the relationship between the degenerated cholinergic system and anesthesia/surgery-induced
B

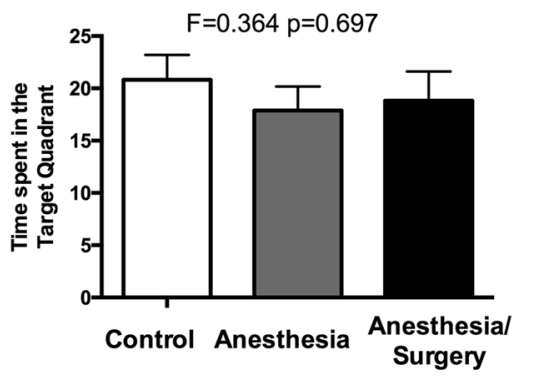

$n=12$, Adult mice
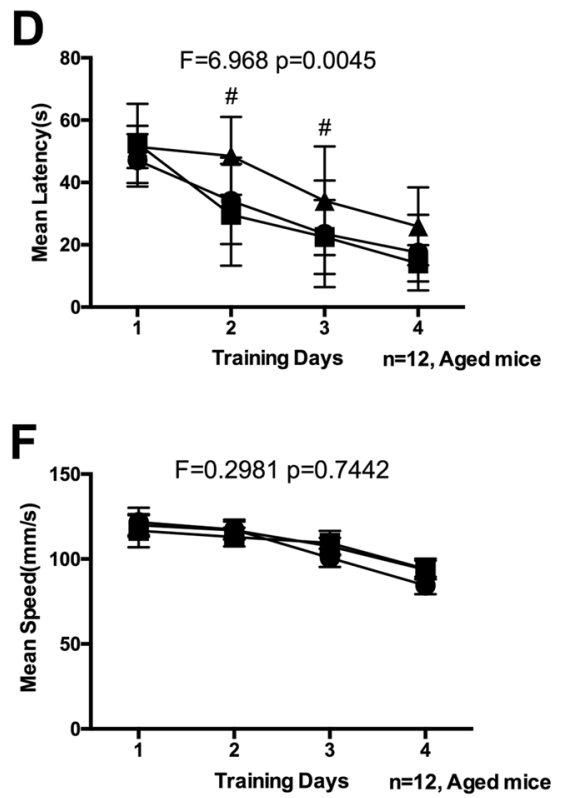

swimming in the target quadrant in every group. The swimming time spent in the target quadrant on the probe tests in anesthesia/surgery group was much shorter than that in the anesthesia and control group. $\mathbf{f}$ There were no differences in the average swimming speed among the three groups (anesthesia/surgery, anesthesia, and control group) in the aged mice. There was no significant difference between the control group and the anesthesia alone group. The data are expressed as the means \pm SEM ( $\# p<0.05$ compared with control group; $* p<0.05$ compared with the target quadrant, $n=12$ per group). MWM Morris water maze

POCD, we injured the central cholinergic system with the mu-p75-sap immunotoxin in adult mice. The effects of mup75-sap at different doses, which ranged from 0.2 to $0.8 \mu \mathrm{g}$, were evaluated in our pilot study, and $0.8 \mu \mathrm{g}$ was identified as the optimal dose that could significantly inhibit hippocampal ChAT levels without affecting cognitive function in adult mice. Therefore, in all subsequent studies, $0.8 \mu \mathrm{g}$ of mu-p75-sap was used in adult mice. Immunohistochemistry and western blot analysis were used to examine the expression levels of the cholinergic biomarkers in the basal 

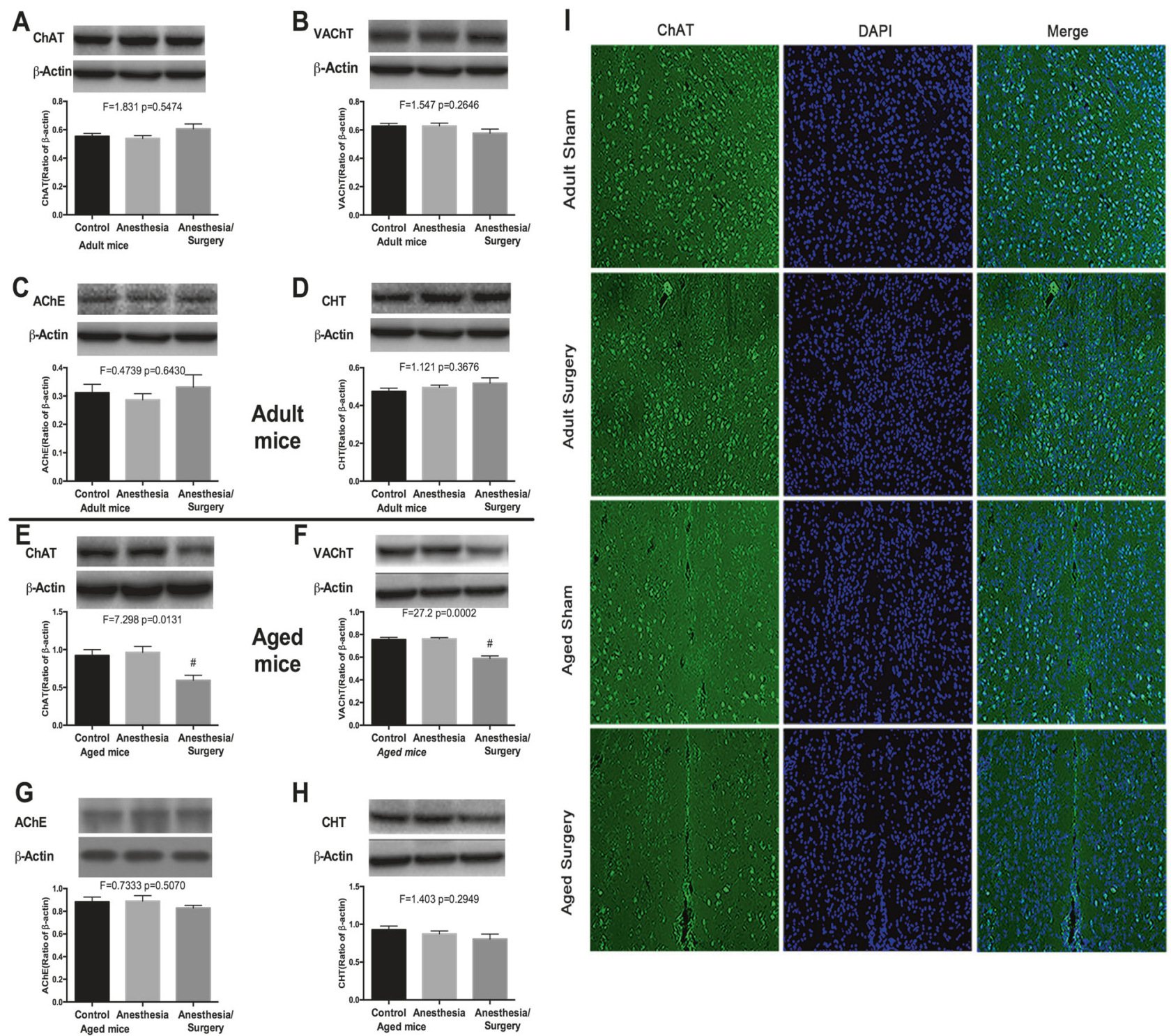

Fig. 2 Anesthesia/surgery decreased the cholinergic biomarkers in the hippocampus of aged but not adult mice. Quantitative western blot showed that there were no differences in the levels of ChAT (a), VAChT (b), AChE (c), or CHT (d) in the hippocampus of adult mice in anesthesia/surgery, anesthesia, and control group. Anesthesia/surgery significantly decreased the levels of ChAT (e) and VAChT (f) level in the hippocampus of the aged mice. There was no significant difference in the levels of $\mathrm{AChE}(\mathbf{g})$ and $\mathrm{CHT}(\mathbf{h})$ in the aged mice in

forebrain after treatment with mu-p75-sap. As shown in Supplemental Fig. 2a-d, $0.8 \mu$ of mu-p75-sap significantly decreased the number of ChAT-positive neurons in the basal forebrain. Results from western blot analysis are consistent with the findings from Immunohistochemical study, showing that $0.8 \mu \mathrm{g}$ of mu-p75-sap significantly decreased the expression of ChAT (Fig. 4a).

Although mu-p75-sap did not affect spatial learning or memory in the adult mice without surgery, it significantly impaired the behavioral performance of the adult mice that underwent anesthesia/surgery. The adult mice that received

the anesthesia/surgery, anesthesia, and control group. i Immunofluorescence analysis of cholinergic neurons in basal forebrain show that cholinergic neurons decreased with aging, and anesthesia/surgery profoundly reduced cholinergic neurons in the aged mice. Scale bar: $100 \mu \mathrm{m}$. The data are expressed as the means $\pm \operatorname{SEM}(n=4$ per group). ChAT choline acetyltransferase, AChE acetylcholinesterase, VAChT vesicular acetylcholine transporter, CHT choline transporter

the combination of mu-p75-sap and anesthesia/surgery spent more time finding the platform and less time in the target quadrant during the probe tests compared with the adult mice that received anesthesia/surgery alone. $(P<0.05$, Fig. 4b, c).

\section{Discussion}

In the present study, we demonstrated that anesthesia/surgery had no effect on the adult mice, but it decreased the 
A

ChAT

$\beta$-Actin
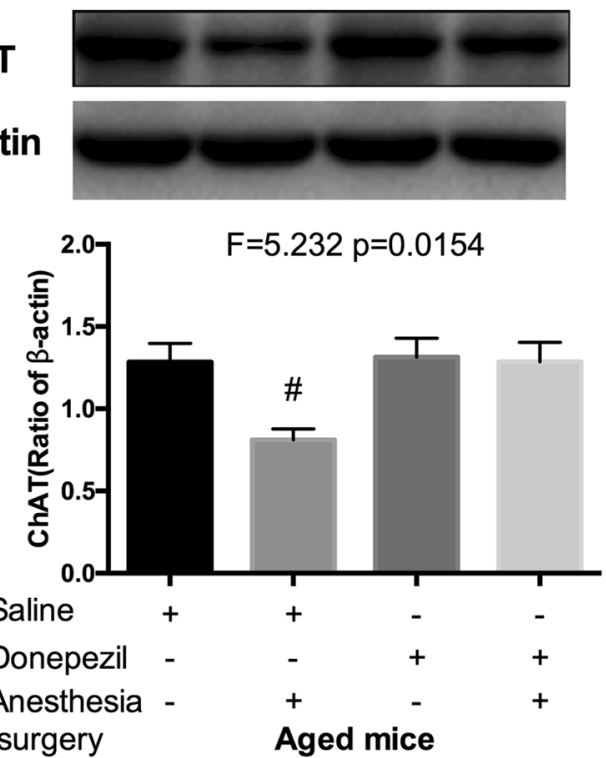

C

\section{AChE}

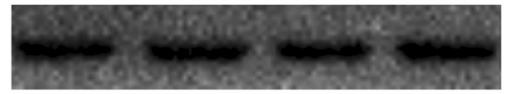

$\beta$-Actin

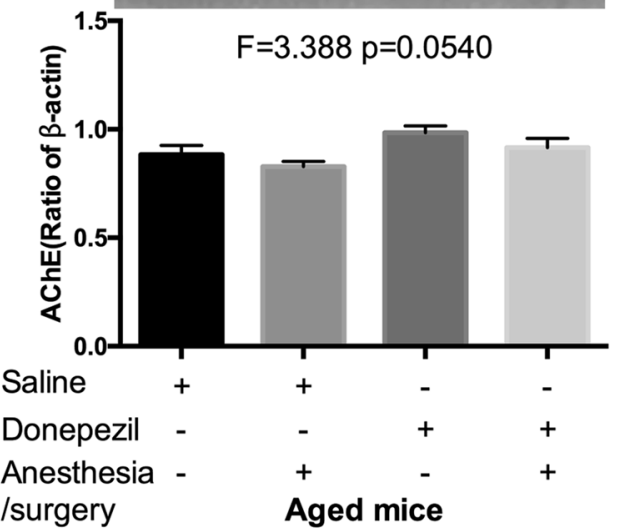

B

VAChT

$\beta$-Actin
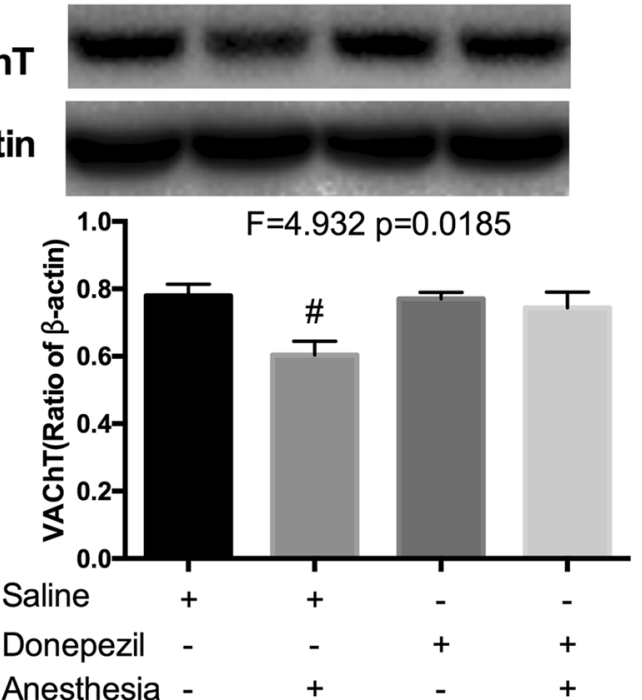

/surgery

D

\section{CHT}

$\beta$-Actin
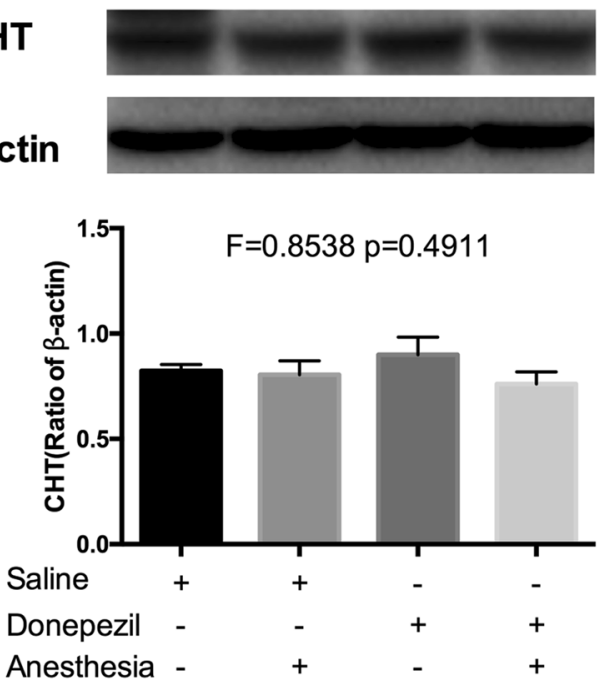

/surgery

Aged mice

E

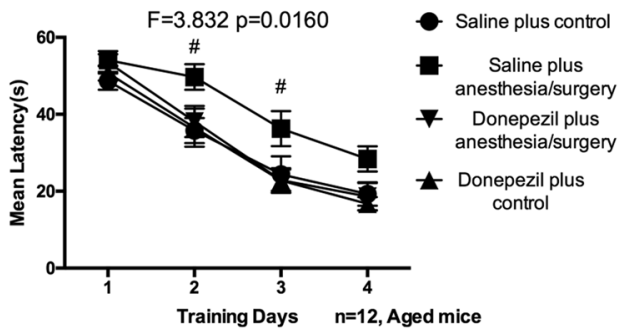

F

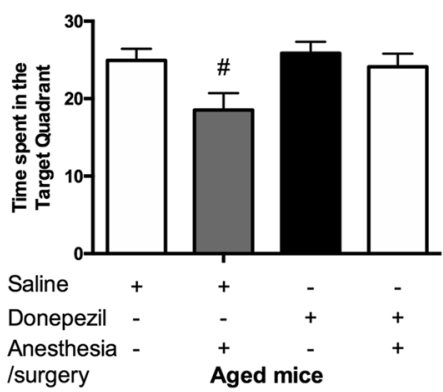

G

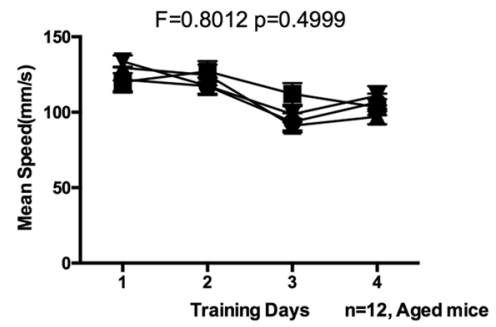

learning and memory ability of aged mice. The expression level of two biomarkers of the central cholinergic system, hippocampal ChAT and VAChT, decreased significantly after anesthesia/surgery in aged but not adult mice. Pretreatment with donepezil, an inhibitor of AChE, prevented cognitive impairment after anesthesia/surgery in aged mice. 
Fig. 3 Donepezil pretreatment prevented the cholinergic biomarker changes and learning and memory impairment induced by anesthesia/ surgery in aged mice. Quantitative western blot analysis showed that anesthesia/surgery significantly decreased the levels of ChAT (a) and VAChT (b) levels as compared with the control condition; donepezil attenuated the anesthesia/surgery-induced reduction in the levels of ChAT (a) and VAChT (b) $(n=4$ per group in a-d). e Anesthesia/ surgery increased the escape latency of MWM test; donepezil mitigated the anesthesia/surgery-induced increase in the escape latency of MWM test. f Anesthesia/surgery decreased the swimming time in the target quadrant in the probe test as compared with control condition; donepezil mitigated the anesthesia/surgery-induced decrease in the target quadrant of MWM test. $\mathrm{g}$ There were no significant differences in the average swimming speed among the four (NS group, anesthesia/ surgery group, Donepezil group, and donepezil + anesthesia/surgery group) groups. ( $n=12$ per group in $\mathrm{E}-\mathrm{G})$. The data are expressed as the means \pm SEM. $\# p<0.05$ compared with NS group; $* p<0.05$ compared with the target quadrant. ChAT choline acetyltransferase, NS normal saline, AChE acetylcholinesterase, VAChT vesicular acetylcholine transporter, $\mathrm{CHT}$ choline transporter

In contrary, pretreatment of mu-p75-sap, an immune toxin specific to cholinergic neurons, induced spatial memory impairment after anesthesia/surgery in adult mice.

Previous studies have shown that aging is a risk factor for postoperative cognitive dysfunction [3, 4, 23].Our previous study also demonstrated that isoflurane exposure impaired the learning and memory of aged mice [18]. However, the reason why aged mice are more easily to develop impairment in learning and memory is not clear yet.

The CNS cholinergic system is widely considered to be essential in learning and memory, including the modulation of the acquisition, encoding, consolidation, reconsolidation, extinction, and retrieval of memory [24]. It degenerates during aging [13] and in neurodegenerative diseases such as AD [24]. Central cholinergic neurons are generally divided into two categories: the first are "interneurons", the majority of which are distributed in the striatum and mainly involved in local neural circuits; the other category consist of "projection neurons", which are distributed mainly in the cholinergic basal forebrain complex and the cholinergic pontine-tegmental of midbrain complex [25-27]. Cholinergic neurons in the basal forebrain project to hippocampus, cortex, olfactory bulb and amygdala [28], modulating memory and hippocampal plasticity [29]; whereas those in the pontine and tegmental nucleus project to the thalamus and brainstem reticular nucleus. Since the hippocampus is an important brain area responsible for learning and memory [30, 31], whose dysfunction has been proven to lead to cognitive impairment [32, 33], the majority of research [34-37] in this area has focused on the hippocampus. Therefore, the present study also focused on cholinergic biomarker changes in the hippocampus and examined changes of cholinergic neurons in basal forebrain, which project to the hippocampus.
In this study, we show that although anesthesia/surgery was stressful to the brain, it had no detectable effect on the normal cholinergic system in non-aged adults. However, it impaired cognitive function in animals with degenerated cholinergic systems, i.e., aged mice or adult mice pretreated with an immune toxin specific to cholinergic neurons. These findings suggest that the level of degeneration of the central cholinergic system before surgery is associated with the development of POCD. Therefore, besides anesthesia and/or surgery, the patients' own characteristics, including age and the status of CNS cholinergic system, are key determinants of POCD development. These findings also suggest that the cholinergic markers, such as acetylcholine, ChAT or AChE, in the CSF or blood of patients who are subjected to surgery, could be used to predict the risk of POCD in the clinic. Actually, a clinical trial examining this possibility is currently ongoing in orthopedics patients (NCT 02063542) in our center. In patients who are identified having reduced central cholinergic function, and could be at increased risk of developing POCD, prophylactic treatments might be especially helpful.

Donepezil is a commercial medication used as the firstline pharmaceutical to treat $\mathrm{AD}$. In the current study, pretreatment of donepezil significantly ameliorated the anesthesia/surgery-induced decrease of hippocampal ChAT and VAChT and prevented the development of POCD, indicating that it might have therapeutic potential for inhibiting POCD in patients. A clinical trial evaluating the clinical effectiveness of donepezil on POCD development is currently ongoing in our hospital (NCT 02927522).

Neuroinflammation might be a key factor in the development of POCD. Maze and colleagues explored a pathway beginning with peripheral surgery, causing peripheral inflammation, then neuroinflammation through a damaged $\mathrm{BBB}$, and ultimately leading to POCD in mice [38, 39]. Our previous study [40] showed that neuroinflammation did occur after surgery, even in adult mice. Rosczyk [7] found that minor surgery results in significant neuroinflammation with an increased level of IL- $1 \beta$ mRNA in the hippocampus of aged mice. Since the central cholinergic system has inhibitory effects on neuroinflammation through a cholinergic anti-inflammation reflex [41], we speculate that a degenerated cholinergic system may not inhibit neuroinflammation effectively, therefore contributing to the development of POCD. Further studies are warranted to test this hypothesis.

$\mathrm{A} \beta$ accumulation in hippocampus has been proposed as another factor in the development of POCD. $\mathrm{Xu}$ [42] demonstrated that abdominal surgery under local anesthesia impaired learning and memory and increased $\mathrm{A} \beta$ levels in the hippocampus of aged mice but not adult mice. There is a complicated interaction between cholinergic system and $\mathrm{A} \beta$ 

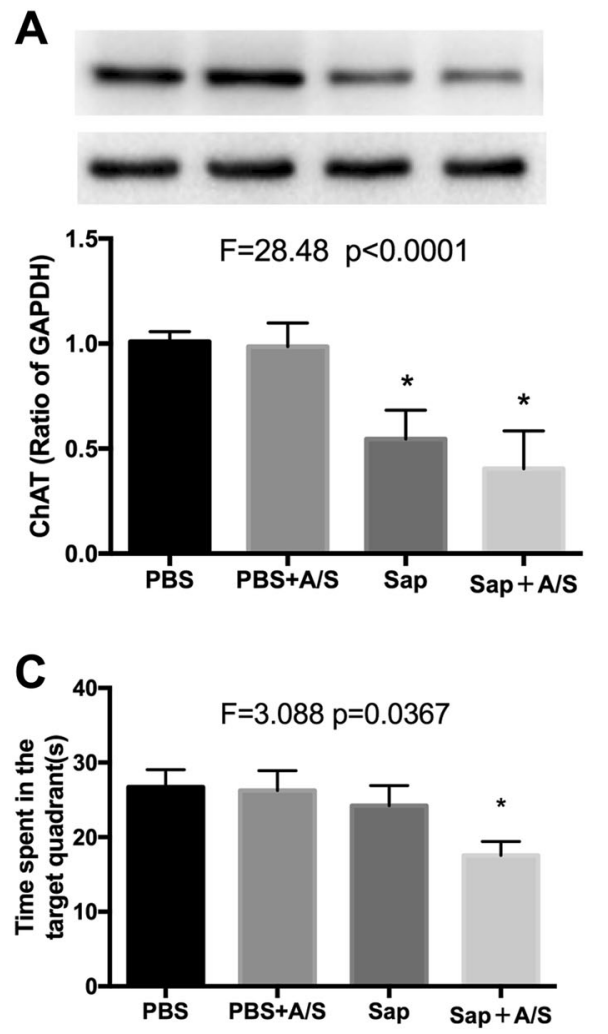

Fig. 4 Spatial learning and memory were impaired after anesthesia/ surgery in adult mice with central cholinergic systems injured by mup75-sap. a Quantitative western blot analysis showed that mu-p75-sap significantly decreased ChAT levels in the hippocampus compared with that in the PBS and PBS $+\mathrm{A} / \mathrm{S}$ group. ChAT level was decreased further in the Sap $+\mathrm{A} / \mathrm{S}$ group, but no statistical significant was found compared with Sap group. b The mean latency in the Sap $+\mathrm{A} / \mathrm{S}$ group significantly increased compared with the PBS group, although A/S

metabolism [43]. Harkany [44] found that $A \beta_{1-42}$ could reduce the ChAT-immunoreactive neurons in the basal forebrain. Muscarinic $\mathrm{m} 1$ - and $\mathrm{m} 3$-receptor activation would stimulate soluble APP $\alpha$ release from rat cortical slices [45]. However, the perioperative interaction of the cholinergic system and $A \beta$ is not clear yet and more studies are warranted.

Our previous study [18] showed that isoflurane exposure impaired the learning and memory of aged mice. Therefore, in the present study, neuroleptic analgesia (fentanyl + droperidol) was utilized to avoid the effects of anesthesia because these medications were proven to have no impact on learning and memory in rodents [15]. All animals tolerated the surgical procedure well and had a full recovery. No mice showed signs of pain after surgery. In accordance with Wan et al. [15], learning and memory ability was not significantly different between the control and the anesthesia groups in the present study, neither were the expression levels of cholinergic markers.
B

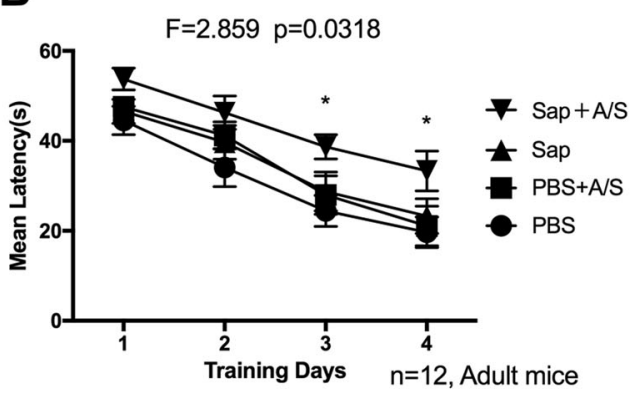

D

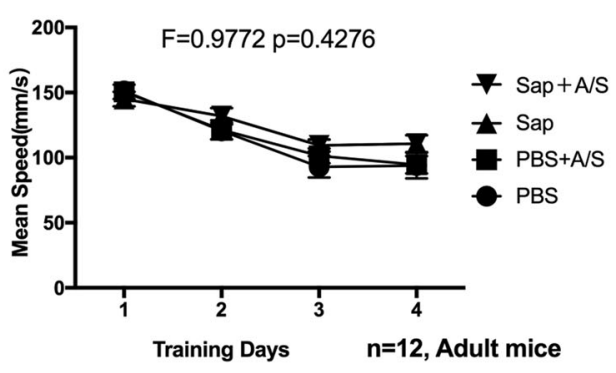

did not affect the latency in these mice. c On the probe test, the swimming time spent in the target quadrant was significantly reduced in mice of Sap $+\mathrm{A} / \mathrm{S}$ group. d There were no differences in the average swimming speed among the four groups. $(n=12$ per group in b-d). The data are expressed as the means \pm SEM. ChAT choline acetyltransferase, PBS phosphate-buffered saline, A/S anesthesia/ Surgery. ${ }^{*} p<0.05$ compared with PBS group

Our study has several limitations. First, although our data demonstrated the important role of the degenerated central cholinergic system in POCD, we do not know why surgery further impaired the degenerated cholinergic system but not the normal system. Further mechanistic studies are currently under investigation in our lab. Secondly, although neuroleptic analgesia, which was utilized in the present study, was not associated with any behavior dysfunction or change of central cholinergic markers, local anesthesia might be a better choice to fully exclude the impacts from anesthesia.

In conclusion, we show that degeneration of central cholinergic neurons, either in aged mice or via mu-p75-sap injection in adult mice, may be important for the development of POCD. The current study may provide insights into mechanisms that underlie an aging-related susceptibility to the development of cognitive dysfunction after surgery. Donepezil pretreatment might be helpful to decrease the risk of POCD. 
Acknowledgements This study was supported by grants from the National Natural Science Foundation of China (No. 81571030, 81771133), Shanghai Pudong New Area Municipal Commission of Health and Family Planning Funding(PW2016D-4), Shanghai Jiao Tong University integration founding of Medicine and Engineering (YG2017MS53), Shanghai Shenkang Hospital Development Center Founding(SHDC12017X11), the Shanghai Municipal Commission of Health and Family Planning Funding for Key Developing Disciplines (2015ZB0101) and the Fundamental Research Funds for the Central Universities(1511219031).

\section{Compliance with ethical standards}

Conflict of interest The authors declare that they have no conflict of interest.

Publisher's note: Springer Nature remains neutral with regard to jurisdictional claims in published maps and institutional affiliations.

\section{References}

1. van Harten AE, Scheeren TW, Absalom AR. A review of postoperative cognitive dysfunction and neuroinflammation associated with cardiac surgery and anaesthesia. Anaesthesia. 2012;67:280-93.

2. Bilotta F, Doronzio A, Stazi E, Titi L, Fodale V, Di Nino G, et al. Postoperative cognitive dysfunction: toward the Alzheimer's disease pathomechanism hypothesis. J Alzheimers Dis. 2010;22 (Suppl 3):81-9.

3. Moller JT, Cluitmans P, Rasmussen LS, Houx P, Rasmussen H, Canet $\mathrm{J}$, et al. Long-term postoperative cognitive dysfunction in the elderly ISPOCD1 study. ISPOCD investigators. International Study of Post-Operative Cognitive Dysfunction. Lancet. 1998;351:857-61.

4. Newman MF, Kirchner JL, Phillips-Bute B, Gaver V, Grocott H, Jones $\mathrm{RH}$, et al. Longitudinal assessment of neurocognitive function after coronary-artery bypass surgery. N Engl J Med. 2001;344:395-402.

5. Steinmetz J, Christensen KB, Lund T, Lohse N, Rasmussen LS. Long-term consequences of postoperative cognitive dysfunction. Anesthesiology. 2009;110:548-55.

6. Steinmetz J, Rasmussen LS. Peri-operative cognitive dysfunction and protection. Anaesthesia. 2016;71(Suppl 1):58-63.

7. Rosczyk HA, Sparkman NL, Johnson RW. Neuroinflammation and cognitive function in aged mice following minor surgery. Exp Gerontol. 2008;43:840-6.

8. Wan Y, Xu J, Meng F, Bao Y, Ge Y, Lobo N, et al. Cognitive decline following major surgery is associated with gliosis, betaamyloid accumulation, and tau phosphorylation in old mice. Crit Care Med. 2010;38:2190-8.

9. Su D, Zhao Y, Wang B, Li W, Xiao J, Chen J, et al. Repeated but not single isoflurane exposure improved the spatial memory of young adult mice. Acta Anaesthesiol Scand. 2011;55:468-73.

10. Schliebs R, Arendt T. The cholinergic system in aging and neuronal degeneration. Behav Brain Res. 2011;221:555-63.

11. Schliebs R, Arendt T. The significance of the cholinergic system in the brain during aging and in Alzheimer's disease. J Neural Transm. 2006;113:1625-44.

12. Fujiki M, Kobayashi H, Uchida S, Inoue R, Ishii K. Neuroprotective effect of donepezil, a nicotinic acetylcholine-receptor activator, on cerebral infarction in rats. Brain Res. 2005;1043:236-41.
13. Perry EK, Johnson M, Kerwin JM, Piggott MA, Court JA, Shaw $\mathrm{PJ}$, et al. Convergent cholinergic activities in aging and Alzheimer's disease. Neurobiol Aging. 1992;13:393-400.

14. Leal T, Lebacq J, Vanbinst R, Lederman C, De Kock M, Wallemacq P. Successful protocol of anaesthesia for measuring transepithelial nasal potential difference in spontaneously breathing mice. Lab Anim. 2006;40:43-52.

15. Wan Y, Xu J, Ma D, Zeng Y, Cibelli M, Maze M. Postoperative impairment of cognitive function in rats: a possible role for cytokine-mediated inflammation in the hippocampus. Anesthesiology. 2007;106:436-43.

16. Krieglstein CF, Cerwinka WH, Laroux FS, Grisham MB, Schurmann G, Bruwer M, et al. Role of appendix and spleen in experimental colitis. J Surg Res. 2001;101:166-75.

17. Yao T, Ying X, Zhao Y, Yuan A, He Q, Tong H, et al. Vitamin D receptor activation protects against myocardial reperfusion injury through inhibition of apoptosis and modulation of autophagy. Antioxid Redox Signal. 2015;22:633-50.

18. Su D, Zhao Y, Wang B, Xu H, Li W, Chen J, et al. IsofluraneInduced Spatial Memory Impairment in Mice is Prevented by the Acetylcholinesterase Inhibitor Donepezil. PLoS ONE. 2011;6: e27632.

19. Shigeta M, Homma A. Donepezil for Alzheimer's disease: pharmacodynamic, pharmacokinetic, and clinical profiles. CNS Drug Rev. 2001;7:353-68.

20. Inanaga K, Ichiki T, Miyazaki R, Takeda K, Hashimoto T, Matsuura $\mathrm{H}$, et al. Acetylcholinesterase inhibitors attenuate atherogenesis in apolipoprotein E-knockout mice. Atherosclerosis. 2010;213:52-8.

21. Saxena G, Singh SP, Agrawal R, Nath C. Effect of donepezil and tacrine on oxidative stress in intracerebral streptozotocin-induced model of dementia in mice. Eur J Pharmacol. 2008;581:283-9.

22. Moreau PH, Cosquer B, Jeltsch H, Cassel JC, Mathis C. Neuroanatomical and behavioral effects of a novel version of the cholinergic immunotoxin mu p75-saporin in mice. Hippocampus. 2008:18:610-22.

23. Vizcaychipi MP, Watts HR, O'Dea KP, Lloyd DG, Penn JW, Wan $\mathrm{Y}$, et al. The therapeutic potential of atorvastatin in a mouse model of postoperative cognitive decline. Ann Surg. 2014;259:1235-44.

24. Ferreira-Vieira TH, Guimaraes IM, Silva FR, Ribeiro FM. Alzheimer's disease: targeting the cholinergic system. Curr Neuropharmacol. 2016;14:101-15.

25. Houser CR, Crawford GD, Salvaterra PM, Vaughn JE. Immunocytochemical localization of choline acetyltransferase in rat cerebral cortex: a study of cholinergic neurons and synapses. J Comp Neurol. 1985;234:17-34.

26. Minces V, Pinto L, Dan Y, Chiba AA. Cholinergic shaping of neural correlations. Proc Natl Acad Sci USA. 2017;114:5725-30.

27. von Engelhardt J, Eliava M, Meyer AH, Rozov A, Monyer H. Functional characterization of intrinsic cholinergic interneurons in the cortex. J Neurosci. 2007;27:5633-42.

28. Gielow MR, Zaborszky L. The Input-output relationship of the cholinergic basal Forebrain. Cell Rep. 2017;18:1817-30.

29. Maurer SV, Williams CL. The Cholinergic system modulates memory and hippocampal plasticity via its interactions with nonneuronal cells. Front Immunol. 2017;8:1489.

30. Rolls ET, Wirth S. Spatial representations in the primate hippocampus, and their functions in memory and navigation. Prog Neurobiol. 2018;171:90-113.

31. Lamsa K, Lau P. Long-term plasticity of hippocampal interneurons during in vivo memory processes. Curr Opin Neurobiol. 2018;54:20-7.

32. Nam SM, Hwang H, Seo M, Chang BJ, Kim HJ, Choi SH, et al. Gintonin attenuates d-galactose-induced hippocampal senescence 
by improving long-term hippocampal potentiation, neurogenesis, and cognitive functions. Gerontology. 2018;64:1-14.

33. Wang W, Duclot F, Groveman BR, Carrier N, Qiao H, Fang XQ, et al. Hippocampal protein kinase D1 is necessary dhpg-induc learn mem impair rats. PLoS ONE. 2018;13:e0195095

34. Netto MB, de Oliveira Junior AN, Goldim M, Mathias K, Fileti $\mathrm{ME}$, da Rosa N, et al. Oxidative stress and mitochondrial dysfunction contributes to postoperative cognitive dysfunction in elderly rats. Brain Behav Immun. 2018;73:661-9.

35. Tajerian M, Hung V, Nguyen H, Lee G, Joubert LM, Malkovskiy $\mathrm{AV}$, et al. The hippocampal extracellular matrix regulates pain and memory after injury. Mol Psychiatry 2018;23:2303-13.

36. Bussian TJ, Aziz A, Meyer CF, Swenson BL, van Deursen JM, Baker DJ. Clearance of senescent glial cells prevents taudependent pathology and cognitive decline. Nature. 2018;562:578-82.

37. Hueston CM, O'Leary JD, Hoban AE, Kozareva DA, Pawley LC, O'Leary OF, et al. Chronic interleukin-1beta in the dorsal hippocampus impairs behavioural pattern separation. Brain Behav Immun. 2018;74:252-64.

38. Cibelli M, Fidalgo AR, Terrando N, Ma D, Monaco C, Feldmann $\mathrm{M}$, et al. Role of interleukin-1beta in postoperative cognitive dysfunction. Ann Neurol. 2010;68:360-8.

39. Terrando N, Monaco C, Ma D, Foxwell BM, Feldmann M, Maze M. Tumor necrosis factor-alpha triggers a cytokine cascade yielding postoperative cognitive decline. Proc Natl Acad Sci USA. 2010;107:20518-22.

40. Zhao Y, Huang L, Xu H, Wu G, Zhu M, Tian J, et al. Neuroinflammation Induced by surgery does not impair the reference memory of young adult mice. Mediators Inflamm. 2016;2016:3271579.

41. Tyagi E, Agrawal R, Nath C, Shukla R. Inhibitory role of cholinergic system mediated via alpha7 nicotinic acetylcholine receptor in LPS-induced neuro-inflammation. Innate Immun. 2010;16:3-13.

42. Xu Z, Dong Y, Wang H, Culley DJ, Marcantonio ER, Crosby G, et al. Age-dependent postoperative cognitive impairment and Alzheimer-related neuropathology in mice. Sci Rep. 2014;4: 3766.

43. Kar S, Slowikowski SP, Westaway D, Mount HT. Interactions between beta-amyloid and central cholinergic neurons: implications for Alzheimer's disease. J Psychiatry Neurosci. 2004;29:427-41.

44. Harkany T, Lengyel Z, Soos K, Penke B, Luiten PG, Gulya K. Cholinotoxic effects of beta-amyloid (1-42) peptide on cortical projections of the rat nucleus basalis magnocellularis. Brain Res. 1995;695:71-5.

45. Pittel Z, Heldman E, Barg J, Haring R, Fisher A. Muscarinic control of amyloid precursor protein secretion in rat cerebral cortex and cerebellum. Brain Res. 1996;742:299-304. 